\title{
Double Adjoints of Hammerstein Functionals Defined on Continuous Functions (*).
}

\author{
ANDRE DE KoRVIN (Terre Haute, Indiana, U.S.A.) \\ Charles E. Roberts, JR. (Terre Haute, Indiana, U.S.A.)
}

\begin{abstract}
Summary. - One of the main reasons for studying Hammerstein operators is their possible application to the analytic study of nonlinear differential equations. In fact, the kernel $\varphi$ used to represent such operators has properties similar to ones that insure the existence of solutions to equations of the form $\dot{x}(t)=\varphi(x(t), t)$ or $\dot{x}(t)=\varphi(x(t))$. The main purpose of the present paper is to study limits of sequences of Hammerstein functionals. While these functionals fail to be linear, their adjoints (in the sense of J. Batt, see [3] and [4]) are linear operators. The Vitali-Hahn-Saks theorem is one of the main tools used to show that if the double adjoint of $T_{n}$ converges on simple functions, then $T_{n}$ converges to $T$ uniformly over an appropriate set of continuous functions. Moreover, $T$ is Hammerstein. Other results use the kernel representation of $T_{n}$ and $T$. This situation is of particular interest when the double adjoints of $T_{n}$ are dominated by probability measures.
\end{abstract}

\section{1. - Introduction.}

Recently many extensions of Riesz type theorems to nonlinear operators have been obtained; see for example [1], [2], [3], [8], and [11]. In [2] operators on $M\left(X, \mathfrak{R}_{)}\right)$ the space of $X$ valued functions that are uniform limits of $\mathscr{B}_{3}$-simple functions are represented, while in [11] non linear operators on $L^{p}$ spaces are considered. Essentially there are two main types of representation: The first type represents the operator as an integral with respect to a positive measure involving an appropriate kernel function, for example $T(f)=\int \varphi[f(t), t] d \mu(t)$ as in [11]. The second type of representation is via an integral with respect to a measure that maps a measurable set into an appropriate non linear operator-such an approach is taken in [1], [2], and $[3]$.

In [3] J. BATT studies operators defined on $O(K, X)$ satisfying

$$
T\left(f+f_{1}+f_{2}\right)=T\left(f+f_{1}\right)+T\left(f+f_{2}\right)-T(f)
$$

whenever $f_{1}$ and $f_{2}$ have disjoint supports. In this case $K$ denotes a compact set and $C(K, X)$ denotes the space of $X$ valued continuous functions (under the sup norm). Operators satisfying (1) were called Hammerstein operators by J. BATT and

(*) Entrata in Redazione il 25 settembre 1979. 
additive operators by FrIEDMAN and TONG [8]. Hammerstein operators have direct applications to the theory of generalized random processes (see [9]) and to the theory of fading memory in continuum mechanics (see [5]). One of the main reasons for studying Hammerstein operators, however, is the possible application to the functional analytic study of non linear differential equations. In fact the kernel $\varphi$ used to represent $T$ as in [11] has properties very similar to the kernel used in the study of the existence of solutions to the equation $\dot{x}(t)=\varphi(x(t), t)$ or the equation $\dot{x}(t)=$ $=\varphi[x(t)]$. For additional references on applications of this type the reader is referred to [11].

The main purpose of the present article is to obtain limit theorems for sequences of Hammerstein functionals. The main technical tool for these results will be the existence of the double adjoint for these functionals. While each functional fails to be linear it has a linear adjoint in the sense of [4]. J. BATT in [4] characterizes the compact and weakly compact mappings among $A$-bounded (not necessarily linear) maps. One of the main points there, is the introduction of the space $E^{\beta}$ in which the adjoint takes values and which plays the role of the traditional dual space $E^{\prime}$. The properties of the double adjoint (for the linear case) was used in [10] where the authors study the w. c. a. property.

Let $T$ be a mapping from $C(K, X)$ into $C$ where $X$ represents a Banach space and $G$ represents the scalar field. Assume that $T$ satisfies (1). It is shown in [3] that under additional continuity conditions $T(f)=\int f d m$ where $m$ maps $\mathscr{B}$ into $M(X, O)$. There $\mathcal{B}$ denotes the Borel field of $K$ and $M(X, C)$ denotes certain maps from $X$ into $C$. Of course, $\int f d m$ is additive in $f$ (but not linear). Our first result is a Egoroff type theorem-that is, if $s_{n}$ is a sequence of simple functions converging $m$ a.e. (this will be defined later) to $f$, then $K$ may be partitioned into a sequence $A_{k}$ and a set $N$ such that $s_{n}$ converges to $f$ uniformly on $A_{k}$ and $\int_{N} s_{n} d m=0$ for all $n$. Next we consider simple functions of the form $\sum \chi_{C_{l}} x_{i}$ where $C_{i}$ are disjoint sets, $\chi_{C_{l}}$ is the characteristic function of $O_{i}$ and $x_{i} \in X$. Naturally $\sum \chi_{C_{i}} x_{i}$ fails to be in $C(K, X)$. The question is "How far removed is it from $C(K, X)$ ?" We show that there exists a map^ that is additive on $M(X, \Re)$ such that $\sum \hat{\chi_{C}} x_{i}$ is in the dual of $M_{H}[C(K, X), C]$ (the space of Hammerstein functionals on $O(K, X)$ satisfying some continuity conditions). Moreover

$$
q_{\alpha}\left(\sum \hat{c_{i}} x_{i}\right) \leqslant \frac{\left\|\Sigma \chi_{\sigma_{i}} x_{i}\right\|}{\alpha}
$$

$q_{\alpha}$ is a family of semi-norms defined on the dual of $M_{H}[C(K, X), C]$. The Egoroff proposition is used to show this. Again, using the Egoroff proposition, we show that if a sequence $s_{n}$ of simple functions converges $m$ a.e. to $f$ then there exists a partition $B_{k}$ and $N$ of $K$ such that $T^{\prime \prime}\left[\chi_{\hat{B} k}\left(s_{n}-f\right)\right]$ converges to 0 and $\int_{N} s_{n} d m=0$.

Here $T^{\prime \prime}$ denotes the double adjoint of $T \in M_{H}[O(K, X), C]$ in the sense of [4]. Our first theorem asserts that if we have a sequence of functionals $T_{n} \in M_{H}[C(K, X), C]$ 
and if the double adjoints of $T_{n}$ behave appropriately, then we can conclude that $T_{n}$ converges to $T \in M_{H}[C(K, X), C]$. This convergence will be made precise later on and will involve functions in the set $C(K, X)$ of the from $V(\cdot) x$ where $x$ is fixed in $X$ and $V$ is a real valued continuous function on $K$ with $0 \leqslant V \leqslant 1$. Next we look at the kernel representation of the functionals in $M_{H}[C(K, X), C]$. This involves the use of a non linear Radon Nikodym type theorem shown in [3]. We can write

$$
T_{n}(f)=\int u_{n}(t, f(t)) d \mu_{n}(t), \quad T(f)=\int u(t, f(t)) d \mu(t)
$$

(where $T$ is as in the first theorem). Moreover, we show that

$$
\int_{B} u_{n}(t, x) d \mu_{n}(t) \rightarrow \int_{B} u(t, x) d \mu(t) \quad \text { uniformly for } \quad\|x\| \leqslant \alpha
$$

where $u$ has the same properties as $u_{n}$. Finally the third theorem yields conditions under which $\mu_{n}$ may be taken equal to $\mu$. These conditions are similar to the ones imposed on dominated operations as defined in [6] where $T$ is dominated by $\mu$ if $|T(f)| \leqslant \int|f| d \mu$ where $\mu$ is a positive measure.

\section{2. - Results.}

We start by establishing some basic notations. Let $K$ be a compact set, $X$ a Banach space, $\mathscr{B}$ the Borel field of $K$ and $O(K, X)$ and $M(X, \mathcal{B})$ as defined previously. Let $u$ be a function from $X$ into $C$. For $\alpha>0, u_{\alpha}$ denotes the restriction of $u$ to the closed $\alpha$-ball of $X$. Let $\left\|u_{\alpha}\right\|=\sup \|u(x)\|$ where the sup is over $\|x\| \leqslant \alpha$. For $\delta>0$ define

$$
D_{\delta} u_{\alpha}=\sup \|u(x)-u(y)\|
$$

where the sup is over $\|x\| \leqslant \alpha,\|y\| \leqslant \alpha,\|x-y\| \leqslant \delta . \quad M(X, C)$ will denote the space of all functions from $X$ into $C$ bounded on the $\alpha$-balls of $X$, uniformly continuous on bounded sets of $X$ and 0 at 0 . That is, if $u \in M(X, C)$, then

$$
\begin{aligned}
& u(0)=0 \\
& \left\|u_{\alpha}\right\|<\infty \quad \text { for } \alpha>0 \\
& \lim _{\delta \rightarrow 0} D_{\delta} u_{\alpha}=0
\end{aligned}
$$

Let $m$ be a finitely additive function from $\mathcal{B}$ into $M(X, C)$. We set $m_{\alpha}(B)=m\left(B_{\alpha}\right)$ and define

$$
s v\left[m_{\alpha}, B\right]=\sup \left\|\sum m\left(B_{i}\right) x_{i}\right\|
$$

where the sup is over partitions $B_{i}$ of $B$ with $\left\|x_{i}\right\| \leqslant \alpha$. 
Also define

$$
s v_{\delta}\left(m_{\alpha}, \boldsymbol{B}\right)=\sup \left\|\sum m\left(B_{i}\right) x_{i}-\sum m\left(B_{i}\right) y_{i}\right\|
$$

where the sup is over partitions $B_{i}$ of $B$ with $\left\|x_{i}\right\| \leqslant \alpha,\left\|y_{i}\right\| \leqslant \alpha,\left\|x_{i}-y_{i}\right\| \leqslant \delta$.

Unless otherwise stated $m$ will denote a finitely additive set function from $\mathfrak{B}$ into $M(X, C)$ with

$$
s v\left[m_{\alpha}, K\right]<\infty \quad \text { and } \quad \lim _{\delta \rightarrow 0} s v_{\delta}\left[m_{\alpha}, K\right]=0
$$

We define $\int \sum \chi_{C_{i}} x_{i} d m=\sum m\left(C_{i}\right) x_{i}$ for disjoint $C_{i}$. By $M_{H}[O(K, X), O]$ we mean the set of all maps $T$ from $O(K, X)$ into $C$ satisfying

$$
T\left(f+f_{1}+f_{2}\right)=T\left(f+f_{1}\right)+T\left(f+f_{2}\right)-T(f)
$$

where $f_{1}$ and $f_{2}$ have disjoint support

$$
\begin{aligned}
& T(0)=0, \\
& \left\|T_{\alpha}\right\|<\infty \quad \text { for all } \alpha>0 \\
& \lim _{\delta \rightarrow 0} D_{\delta} T_{\alpha}=0 .
\end{aligned}
$$

$Y^{\prime}$ will denote the dual of the space $Y$ and $T^{\prime}$ will denote the adjoint of $T$ (in the sense of [4]). Let $T \in M_{H}[C(K, X), C]$ and let $m_{T}$ be its representative measure-that is, $T(f)=\int f d m_{T}$. It is shown in [3] that $m_{T^{\prime}}$ satisfies the properties stated above for $m$.

Finally we define a property to hold $m$ a.e., if there exists a set $A$ such that the property is true for all $x \notin A$ and for $B \in \mathfrak{B}$ and $B \subset A$ we have $m(B)=0$.

Propostrion (Egoroff). - Assume that $\left\{s_{n}\right\}$ is a sequence of $X$-valued simple functions converging pointwise to $f$. Then there exists a partition of $K$ into sets $A_{k} \in \mathfrak{B}$ and $N \in \mathfrak{B}$ such that

(1) $\left\{s_{n}\right\}$ converges to $f$ uniformly on each $A_{k}(k=1,2, \ldots)$;

(2) $\int_{N} s_{n} d m_{K}=0$ for all $n$.

Proof. - Let

$$
\hat{m}(E)=\sum_{n=1}^{\infty} \frac{\int_{E} s_{n} d m_{T}}{2^{n}\left[1+\sup _{A \in \mathcal{S}}\left|\int_{\mathcal{A}} s_{n} d m_{T}\right|\right]}
$$

clearly $\hat{m}$ is finitely additive and bounded. Moreover since $m_{K}(\cdot) x$ is countably additive for each $x$ (see [3]) it follows that $\hat{m}$ is a bounded countably additive 
measure. Thus since $s_{n}$ converges to $f \hat{m}$ a.e. it follows from the Egoroff theorem [6, p. 95] that there exists sets $A_{k} \in \mathscr{B}$ and $N \in \mathscr{B}$ such that $s_{n}$ converges to $f$ uniformly on $A_{k}$ and $N$ is a $\hat{m}$ null set i.e. $\int_{N} s_{n} d m_{T}=0$.

Note. - The above proof goes through if pointwise convergence is replaced by $m_{T}$ a.e. convergence.

We now define some semi-norms of interest. For every $\alpha>0$ define $p_{\alpha}$ on

$$
M_{H}[C(K, X), O] \quad \text { by } \quad p_{\alpha}(T)=\left\|T_{\alpha}\right\|
$$

Then we can define the dual topology on $M_{H}[C(K, X), C]^{\prime}$ by

$$
q_{\alpha}(V)=\sup |\langle V, T\rangle|
$$

where the sup is over $p_{\alpha}(T) \leqslant 1$ and $V \in M_{H}[C(K, X), C]^{\prime}$. Let $T \in M_{H}[C(K, X), C]$. Since $\left\|T_{\alpha}\right\|<\infty$, the adjoint of $T$ exists by [4]. (Here $\mathcal{A}$ consists of all $\alpha$-balls of $C(K, X)$ and $E^{\mathcal{B}}$ is $M_{H}[C(K, X), C]$ while $E$ is $C(K, X)$.) Let $T^{\prime}$ be the adjoint of $T$

Corollary 1. - There exists a map ^ from $M(X, \mathfrak{B})$ into $M_{H}[C(K, X) \text {, } C]^{\prime}$ that is additive on functions of disjoint supports in $M(X, \mathfrak{B})$ and has the following property :

$$
q_{\alpha}\left(\sum \hat{\chi_{c_{i}}} x_{i}\right) \leqslant \frac{\left\|\sum \chi_{c_{i}} x_{i}\right\|}{\alpha}
$$

where $C_{i}$ are disjoint sets of $\mathfrak{B}$.

Proof. - Let $s_{n}$ be a sequence of $X$-valued simple functions converging to $f$ pointwise. Since $T$ maps bounded sets into bounded sets $T$ is a $A$-weakly compact in the terminology of [4]. Thus $T^{\prime \prime}$ maps $C$ into $M_{H}[C(K, X), C]$ and $T^{\prime \prime}$ maps $M_{H}[C(K, X), C]^{\prime}$ into $C$ (Theorem 1 of [4]). Let $N$ and $B_{k}$ be sets as in the previous proposition. Then $\chi_{B_{k}} s_{n}$ converges uniformly to $\chi_{B_{k}} f$. Thus $\chi_{B_{k}} f \in M(X, \mathfrak{3})$. Let $x \in X$ and $B \in \mathcal{B}$. Every element in $M_{H}[C(K, X), C]$ is of the form $m_{A}$ where $A \in M_{H}[C(K$, $X), C]$ with $A(f)=\int f d m_{A}$. Define $\hat{\chi_{B}} x$ by

$$
\left\langle\hat{\chi_{B}} x, m_{A}\right\rangle=m_{A}(B) x
$$

It is easy to see that $\hat{\chi_{B}} x$ is a linear functional on $M[C(K, X), C]$. Let $p_{\alpha}(A) \leqslant 1$. Since

$$
\chi_{B} x=\frac{\|x\|}{\alpha} \chi_{B} \frac{x}{\|x\|} \alpha,
$$

we can assume without loss of generality that $\|x\| \leqslant \alpha$. Then

$$
\left|\left\langle\hat{\chi_{B}} x, m_{A}\right\rangle\right|=\left|m_{A}(B) x\right| \leqslant s v_{\alpha} m_{A}=p_{\alpha}(A) \leqslant 1 \text {. }
$$

7 - Annali di Matematica 
Thus

$$
\hat{\chi_{B}} \tilde{x} \in \hat{M_{H}}[C(K, X), X]^{\prime}
$$

(It is important to recall that in [3] it has been shown that if $A$ and $m_{A}$ correspond, then $\left.s v_{\alpha} m_{A}=\left\|A_{\alpha}\right\|.\right)$ It is easy to see that ${ }^{{ }^{\prime}}$ is additive on simple functions with disjoint support and hence on $M(X, \Re)$. Of course if $h \in M(X, \mathfrak{B})$, then

Now

$$
\left\langle\hat{\chi_{B}} h, m_{A}\right\rangle=\int_{B} h d m_{A} .
$$

$$
\begin{aligned}
\left|\left\langle\sum_{i} \frac{\left\|x_{i}\right\|}{\alpha} \chi_{c_{i}} \frac{\alpha x_{i}}{\left\|x_{i}\right\|}, m_{A}\right\rangle\right|=\mid \sum_{i} \frac{\left\|x_{i}\right\|}{\alpha} m_{A}\left(C_{i}\right)\left(\frac{\alpha x_{i}}{\left\|x_{i}\right\|} \mid\right) \\
\leqslant \frac{\operatorname{Max}\left\|x_{i}\right\|}{\alpha} s v_{\alpha} m_{A} \leqslant \frac{\left\|\chi_{c_{i}} x_{i}\right\|}{\alpha} \quad \text { if } s v_{\alpha} m_{A} \leqslant 1 .
\end{aligned}
$$

Thus

$$
q_{\alpha}\left(\sum \hat{\chi_{c_{i}}} x_{i}\right) \leqslant \frac{\left\|\sum \chi_{c_{i}} x_{i}\right\|}{\alpha}
$$

Corollary 2. - Let $s_{n}$ be a sequence of $X$ simple functions where $s_{n}$ converges pointwise to $f$. Let $T \in M_{H}[C(K, X), C]$. Then there exists a disjoint family of sets in $\Re, N$ and $\left\{B_{k}\right\}$ such that

(1) $T^{\prime \prime}\left[\hat{{x_{k}}_{B_{n}}}\left(s_{n}-f\right)\right]$ converges to 0 (as $\left.n \rightarrow \infty\right)$ and

(2) $\int_{N} s_{n} d m_{T}=0$ for all $n$.

Proof. - Let $N$ and $B_{k}$ be as earlier. $\chi_{B_{k}} s_{n}$ converges uniformly to $\chi_{B_{k}} f \in M(X, \mathfrak{B})$ (previous corollary). For $k$ fixed,

$$
\left\langle\chi_{B_{k}}\left(\hat{s}_{n}-f\right), m_{A}\right\rangle=\left|\int_{B_{k}}\left(s_{n}-f\right) d m_{A}\right| \leqslant \delta v_{\delta}\left(m_{A}\right)_{\alpha}
$$

where $\left\|\chi_{B_{k}} s_{n}\right\| \leqslant \alpha,\left\|\chi_{B_{k}} f\right\| \leqslant \alpha,\left\|\chi_{B_{k}}\left(s_{n}-\right)\right\| \leqslant \delta$.

Since $m_{A}$ has the property that $s v_{\delta}\left(m_{A}\right)_{\alpha}$ converges to 0 as $\delta \rightarrow 0$ it follows that $\chi_{B_{k}} s_{n}$ converges to $\chi_{B_{k}} f$ in the topology of $M_{H}[C(K, X), X]^{\prime}$. Since $T$ is $A$ weakly compact it follows from [4] that $T^{\prime}$ is $\sigma\left(F^{\prime}, F\right), \sigma M_{H}[C(\dot{K}, X), C], M_{H}[C(K, X), O]^{\prime}$ continuous. Since $T^{\prime}$ is linear it follows (see [12]) that $T^{\prime \prime}$ is weakly continuous and the corollary follows. Again the proof goes through if pointwise convergence is replaced by $m_{n}$ a.e.

We now introduce some new notation. If $B \in \Re$ then $\pi_{0}(B)$ will denote the collection of all compact subsets of $B$ ordered by $K_{1} \leqslant K_{2}$ if $K_{1} \subset K_{2} . \quad \pi(B)$ will denote the collection of all open sets containing $B$ and ordered by $G_{1} \leqslant G_{2}$ if $G_{2} \subset G_{1}$. 
$V\left(C, G^{\prime}\right)$ denotes a function continuous on $K, 1$ on the compact set $C, 0$ off $G$ where $G$ is open and $C \subset G$ and $0 \leqslant v\left(C, G^{\prime}\right) \leqslant 1$. We introduce another family of semi-norms on $M_{H}[C(K, X), C]$ by

$$
p_{\alpha}^{\delta}(T)=D_{\delta} T_{\alpha} \quad \text { for all } \delta>0 \text { and } \alpha>0 .
$$

THEOREM 1. - Let $T_{n}$ be a sequence in $M_{H}[C(K, X), C]$ such that for all $B \in \mathscr{B}$ $T_{n}^{\prime \prime}\left(\hat{\chi_{B}} x\right)$ converges uniformly in $x$ for $\|x\| \leqslant \alpha$. Assume $\sup _{n} p_{\alpha}\left(T_{n}\right)<\infty$ and $\lim _{\delta \rightarrow 0} p_{\alpha}^{\delta}\left(T_{n}\right)=0$ uniformly in $n$. Then there exists $T \in M_{H}[C(K, X), O]$ such that

$$
\lim _{C \in \pi_{0}(B)} \lim _{G \in \pi(C)}\left(T_{n}-T\right)\left[V\left(C, G^{\prime}\right) x\right]=0
$$

uniformly in $\|x\| \leqslant \alpha$. In addition if $B_{l} \in \mathfrak{B}$ and $B_{l} \searrow \varphi$ then

$$
\sup _{n} \sup _{\|x\| \leqslant \infty} \lim _{C \in \pi_{0}\left(B_{l}\right)} \lim _{G \in \pi(C)} T_{n}\left[V\left(C, G^{\prime}\right) x\right]
$$

converges to 0 as $l \rightarrow \infty$.

Proof. - Let $m_{n}$ represent $T_{n}$. By [3] the $\left(m_{n}\right)_{\alpha}$ are countably additive measures. The $\left(m_{n}\right)_{\alpha}$ may be viewed as vector messures into the completion of $M(X, C) / \operatorname{ker} r_{\alpha}$ where $r_{\alpha}$ is a semi norm on $M(X, C)$ defined by

$$
r_{\alpha}(W)=\sup _{\|x\| \leqslant \alpha}|W(x)|
$$

For every $\alpha>0$ let $w_{n, \alpha}$ be a positive measure such that

$$
w_{n, \alpha}(A) \leqslant\left|\left(m_{n}\right)_{\alpha}(A)\right| \quad \text { where } \quad\left|\left(m_{n}\right)_{\alpha}(A)\right|=r_{\alpha} m_{n}(A)
$$

and where in addition $\left(m_{n}\right)_{\alpha} \ll w_{n, \alpha}$ (see [7], p. 321).

Let

$$
w_{\alpha}(A)=\sum_{n} \frac{w_{\alpha, n}(A)}{2^{n}\left[1+w_{\alpha, n}(K)\right]}
$$

Now by [3] $\left(m_{n}\right)_{\alpha}$ are regular measures, thus $w_{\alpha, n}$ are regular and hence $w_{\alpha}$. Using the previous notations we now show that $T^{\prime \prime}\left[\hat{\chi_{B}} x\right]=m_{T^{\prime}}(B) x$. Let $a \in C$ then

$$
\left\langle T^{\prime \prime}\left[\hat{\chi_{B}} x\right], a\right\rangle=\left\langle\hat{\chi_{B}} x, T^{\prime}(a)\right\rangle=m_{T^{\prime}(a)}(B) x .
$$

Now

$$
\begin{aligned}
\left\langle m_{T}(B) x, a\right\rangle & =\lim _{C \in \Pi_{0}(B)} \lim _{G \in \Pi(C)}\left\langle T\left[V\left(C, G^{\prime}\right) x\right], a\right\rangle \\
& =\lim _{G \in \Pi_{\mathbf{o}}(B)} \lim _{G \in \Pi(C)}\left\langle V\left(C, G^{\prime}\right) x, T^{\prime}(a)\right\rangle \\
& =\lim _{C \in \Pi_{\mathbf{o}}(B)} \lim _{G \in \Pi(C)} \int v\left(C, G^{\prime}\right) x d m_{T^{\prime}(a)} .
\end{aligned}
$$

By regularity of $m_{T^{\prime}(a)}$ this is $m_{T^{\prime}(a)}(B) x$. 
Thus,

$$
T^{\prime \prime}\left[\hat{\chi_{B}} x\right]=m_{T}(B) x .
$$

Since $T_{n}^{\prime \prime}\left[\hat{\chi_{B}} x\right]$ converges uniformly for $\|x\| \leqslant \alpha$ it follows that $\left(m_{n}\right)_{\alpha}(B)$ converge in the $r_{\alpha}$ semi-norms. By the Vitali-Hahn-Saks theorem $\left(m_{n}\right)_{\alpha}$ are uniformly absolutely continuous with respect to $w_{\alpha}$ : (Hence $\left(m_{n}\right)_{\alpha}$ are regular and also uniformly countably additive.) Let $m(B)=\lim m_{n}(B)$ in the $r_{\alpha}$ topology. Again by the Nikodym theorem $(m)_{\alpha}$ is countably additive (and regular). Now by the Nikodym boundedness theorem $r_{\alpha}\left[m_{n}(B)\right]$ is uniformly bounded in $n$, say

$$
\sup _{n} r_{\alpha}\left[m_{n}(B)\right] \leqslant K_{\alpha}(B) \quad \text { so } \quad r_{\alpha} m(B) \leqslant r_{\alpha}\left[m(B)-m_{n}(B)\right]+K_{\alpha}(B)
$$

If we pick $n$ so that $r_{\alpha}\left[m(B)-m_{n}(B)\right]<1$ then

$$
\sup _{\|x\| \leqslant a}|m(B) x| \leqslant K_{\alpha}(B)+1 \text {. }
$$

Since $s v_{\alpha}\left(m_{n}\right)=p_{\alpha}\left(T_{n}\right)$ from the finiteness of $\sup _{n} s v_{\alpha}\left(m_{n}\right)$ it follows that since

$$
\left|\sum m\left(B_{i}\right) x_{i}\right| \leqslant\left|\sum\left(m\left(B_{i}\right)-m_{n}\left(B_{i}\right)\right) x_{i}\right|+\left|\sum m_{n}\left(B_{i}\right) x_{i}\right|
$$

then $s v_{\alpha}(m)<\infty$.

Finally,

$$
\begin{aligned}
\left|\sum m\left(B_{i}\right) x_{i}-\sum m\left(B_{i}\right) x_{i}^{\prime}\right| \leqslant \mid \sum & m\left(B_{i}\right) x_{i}-\sum m_{n}\left(B_{i}\right) x_{i} \mid+ \\
& +\left|\sum m_{n}\left(B_{i}\right) x_{i}-\sum m_{n}\left(B_{i}\right) x_{i}^{\prime}\right|+\left|\sum m_{n}\left(B_{i}\right) x_{i}^{\prime}-\sum m\left(B_{i}\right) x_{i}^{\prime}\right|
\end{aligned}
$$

which implies $\lim _{\delta \rightarrow 0} s v_{\delta}(m)_{\alpha}=0$, since the middle term is $p_{\alpha}^{\delta}\left(T_{n}\right)$ and is assumed to $c_{\text {onverge to } 0}$ uniformly in $n$. By [3] it follows that there exists $T \in M_{H}[C(K, X), O]$ $\mathrm{f}_{\text {or }}$ which $m_{T}=m$. Also

$$
\lim _{C \in \Pi_{0}(B)} \lim _{G \in \Pi(C)}\left(T_{n}-T\right)\left[V\left(C, Q^{\prime}\right) x\right]=\left|\left(m_{n}(B)-m(B)\right) x\right|
$$

and this converges to 0 in the $r_{\alpha}$ semi-norms i.e. uniformly as $\|x\| \leqslant \alpha$. Finally let $B_{l} \downarrow \varphi$, then $r_{\alpha} m_{n}\left(B_{l}\right)$ converges to 0 uniformly in $n$, by uniform countable additivity. Thus sup sup $\left|m_{n}\left(B_{l}\right) x\right|$ converges to 0 .

$n$ : $\|x\| \leqslant \alpha$

Let $(S, \Re, \tau)$ be a finite measure space. Let $v$ be a map from $S$ into $M(X, O)$ and let $\varrho$ be a lifting of $L^{\infty}(\tau)$, see [6]. $v$ is called a $K$-kernel if

(1) $v(\cdot, x): t \rightarrow v(t)(x)$ is in $L^{\infty}(\tau)$ for each $x$;

(2) $v(\cdot, x)=\varrho v(\cdot, x)$ for each $x$; 
(3) $t \rightarrow \sup _{\|x\| \leqslant \alpha}|v(t, x)|$ is bounded for each $\alpha>0$;

(4) $\sup _{\|x\| \leqslant \alpha}|v(t)(x)|$ and $D_{\delta} v(\cdot)_{\alpha}: t \rightarrow D_{\delta} v(t)_{\alpha}$ are $\mathcal{B}$ measurable and $\lim _{\delta \rightarrow 0} D_{\delta} v(t)_{\alpha}=0, \tau$ a.e.

THEOREM 2. - Let $T_{n}$ be a sequence in $M_{H}[C(K, X), C]$ satisfying the properties of the previous theorem. Then there exists finite positive measures $\mu_{n}$ and $\mu$ such that

$$
\begin{aligned}
T_{n}(f) & =\int u_{n}(t, f(t)) d \mu_{n}(t) \\
T(f) & =\int u(t, f(t)) d \mu(t)
\end{aligned}
$$

( $T$ is as in the previous theorem) where $u_{n}$ and $u$ are $K$-kernels. Moreover

$$
\int_{B} u_{n}(t, x) d \mu_{n}(t) \text { converges to } \int_{B} u(t, x) d \mu(t)
$$

uniformly for $\|x\| \leqslant \alpha$.

Proof. - Let $m_{n}$ and $m$ represent $T_{n}$ and $T$. It was shown that $m_{n}(B)$ converges to $m(B)$ in the $r_{\alpha}$ semi-norms. Since the variation dominates the semi-variation and is dominated by four times the semi-variation (and the same is true for the $\delta$-variation) it follows that

$$
\sup v\left[\left(m_{n}\right)_{k}\right]<\infty \quad \text { and } \quad \lim _{\delta \rightarrow 0} v_{\delta}\left(m_{n}\right)_{k}=0
$$

where $v$ and $v_{\delta}$ denote the variation and the $\delta$ variation.

Let

$$
\mu_{n}(B)=\sum_{k} \frac{1}{2^{k}} \frac{v\left(m_{n}\right)_{k}(B)}{\left[1+v\left(m_{n}\right)_{k}\right]}
$$

Then $\left(m_{n}\right)_{\alpha}$ is countably additive, $v\left(m_{n}\right)_{\alpha}<\infty, \lim _{\delta \rightarrow 0} v_{\delta}\left(m_{n}\right)=0$ uniformly in $n$ and

$$
v\left(m_{n}\right)_{\alpha}(B) \leqslant L_{\alpha}^{n} \mu_{n}(B)
$$

where $L_{\alpha}^{n}$ is some constant. By [3] there exists $K$-kernels $u_{n}$ such that

$$
T_{n}(f)=\int u_{n}(t, f(t)) d \mu_{n}(t)
$$

and similarly

$$
T(f)=\int u(t, f(t)) d \mu(t)
$$


Since

$$
m_{n}(B) x=\int_{B} u_{n}(t, x) d \mu_{n}(t) \quad \text { and } \quad m(B) x=\int_{B} u(t, x) d \mu(t)
$$

the theorem follows.

The last theorem states that one can pick $\mu_{n}=\mu$ provided the double adjoints of $T_{n}$ are dominated by probability functions.

THEOREM 3. - Let $T_{n}$ be a sequence in $M_{H}[C(K, X), C]$ such that for every $B \in \mathfrak{B}$, $T_{n}^{\prime \prime}\left(\hat{\chi_{B}} x\right)$ converges uniformly for $\|x\| \leqslant \alpha$. Assume that $\lim _{\delta \rightarrow 0} p_{\alpha}^{\delta}\left(T_{n}\right)=0$ uniformly in $n$ and that for every positive integer $k$ there exists probabilities $p_{k}$ such that

$$
\sup _{\|x\| \leqslant k}\left|T_{n}^{\prime \prime}\left(\hat{\chi_{B}} x\right)\right| \leqslant L_{k}^{\prime} p_{k}(B) \quad \text { for } B \in \mathfrak{B} .
$$

Then the conclusion of the previous theorem hold with $\mu_{n}=\mu$.

$$
\text { Proof. - Let }
$$

$$
\mu(B)=\sum_{k} \frac{p_{k}(B)}{\left[1+p_{k}(K)\right] 2^{k}} .
$$

From the hypothesis it follows that $v\left(m_{n}\right)_{k}(B) \leqslant L_{k}^{\prime} p_{k}(B) \leqslant L_{k} \mu(B)$.

The proof then proceeds as in Theorem 2.

\section{BIBLIOGRAPHY}

[1] R. ALo - A. DE Konvin, Representation of Hammerstein operators by Nemytskii measures, J. of Math. Anal. and Appl., 152 (1975), pp. 490-513.

[2] R. Alo - A. DE Konvin - Vo VAN Tho, Integration theory for Hammerstein operators, J. of Math. Anal. and Appl., 51 (1977), pp. 72-96.

[3] J. BATt, Nonlinear integral operators on $C(S, E)$, Studia Math., 48 (1973), pp. 145-177.

[4] J. BAтT, Nonlinear compact mappings and their adjoints, Math. Ann., 189 (1970), pp. 5-25.

[5] B. D. Colmana - V. J. Mrzel, Norms and semi-groups in the theory of Fading memory, Arch. Rat. Mech. Anal., 23 (1966), pp. 87-123.

[6] N. Dinculeanu, Vector Measures, Pergamon Press, Berlin, 1967.

[7] N. Dunford - J. T. Schwartz, Linear operators. I: General theory, Pure and Appl. Math. VII, Interscience, New York, 1958.

[8] N. A. Friedman - A. E. Tong, On additive operators, Canad. J. of Math., 23 (1971), pp. $468-480$.

[9] I. M. GELFAND - N. YA. VILENKIN, Generalized Functions, vol. 4: Applications of Harmonic Analysis, Academic Press, New York (1964), pp. 273-278.

[10] A. DE KORVIN - L. Kuxes, Some non-weak integrals defined by linear functionals, The Quart. J. of Math., 24 (1973), pp. 289.299.

[11] V. J. MIzEL, Characterization of nonlinear transformations possessing kernels, Canad. J. of Math., 22 (1970), pp. 449-471.

[12] A. P. Robertson - W. J. Robertson, Topological Vector Spaces, Cambridge University Press, London, 1964. 